\title{
$R$-TYPE SUMMABILITY METHODS, CAUCHY CRITERIA, $P$-SETS AND STATISTICAL CONVERGENCE
}

\author{
JEFF CONNOR
}

(Communicated by Andrew M. Bruckner)

\begin{abstract}
A summability method $S$ is called an $R$-type summability method if $S$ is regular and $x y$ is strongly $S$-summable to 0 whenever $x$ is strongly $S$-summable to 0 and $y$ is a bounded sequence. Associated with each $R$ type summability method $S$ are the following two methods: convergence in $\mu$-density and $\mu$-statistical convergence where $\mu$ is a measure generated by $S$. In this note we extend the notion of statistically Cauchy to $\mu$-Cauchy and show that a sequence is $\mu$-Cauchy if and only if it is $\mu$-statistically convergent. Let $W(A)=\bar{A}^{\beta \mathbb{N}} \cap \beta \mathbb{N} \backslash \mathbb{N}$ for $A \subset \mathbb{N}$ and $\mathscr{K}=\bigcap\left\{W(A): A \subseteq \mathbb{N}, \chi_{A}\right.$ is strongly $S$-summable to 1 \}. Then $\mu$-Cauchy is equivalent to convergence in $\mu$-density if and only if every $G_{\delta}$ that contains $\mathscr{K}$ in $\beta \mathbb{N} \backslash \mathbb{N}$ is a neighborhood of $\mathscr{K}$ in $\beta \mathbb{N} \backslash \mathbb{N}$. As an application, we show that the bounded strong summability field of a nonnegative regular matrix admits a Cauchy criterion.
\end{abstract}

In this note we explore a variety of structures related to the bounded strong summability field of an $R$-type summability method. We also show, given an $R$ type summability method $S$, how to construct a measure $\mu$ associated with $S$ and define $\mu$-statistical convergence and convergence in $\mu$-density. These methods are, respectively, stronger and weaker than strong $S$-summability on the bounded sequences. We then characterize the measures for which $\mu$-statistical convergence and convergence in $\mu$-density are equivalent. This characterization is given in the context of measures, ideals of bounded sequences, subsets of $\beta \mathbb{N} \backslash \mathbb{N}$, and lattices of summability methods. We also establish a Cauchy criterion for $\mu$-statistical convergence which, via the above characterization, yields a Cauchy criterion for the strong summability of a bounded sequence with respect to a nonnegative regular matrix summability method.

In the following, we let

$$
\begin{aligned}
\omega & =\text { the collection of all real valued sequences, } \\
\varphi & =\{x \in \omega: x \text { is finitely nonzero }\} \\
c_{0} & =\{x \in \omega: \lim x=0\} \\
c & =\{x \in \omega: \lim x \text { exists }\} \\
l_{\infty} & =\left\{x \in \omega: \sup _{n}\left|x_{n}\right|<\infty\right\}
\end{aligned}
$$

Received by the editors March 20, 1990.

1991 Mathematics Subject Classification. Primary 40D20, 40D25.

Key words and phrases. Strong summability, statistical convergence, measures, additive property for null sets.

Research supported by an Ohio University Research Council Grant. 
Let $\|x\|=\sup _{n}\left|x_{n}\right|$ whenever $x \in l_{\infty}$ and let $l_{\infty}$ have the metric topology induced by \|\| . If $x \in \omega$ and $L$ is a scalar, we let $x-L$ denote the sequence $\left(x_{k}-L\right)$ and $|x|$ denote the sequence $\left(\left|x_{k}\right|\right)$.

Following Freedman and Sember [10], we define a summability method to be a linear subspace $c_{S} \subseteq \omega$ and a linear functional $S: c_{S} \rightarrow R$. If $S$ is a summability method we say that a sequence is $S$-summable to $L$ if $S(x)=L$ and we say that:

(1) $S$ is regular if $c \subseteq c_{S}$ and $S(x)=\lim x$ for all $x \in c$.

(2) A sequence $x$ is strongly $S$-summable to $L$ if $S(|x-L|)=0$.

(3) $S$ is an $R$-type summability method (or RSM) if $S$ is regular and $x y$ is strongly $S$-summable to 0 whenever $x$ is strongly $S$-summable to 0 and $y$ is a bounded sequence.

We also let

$$
\begin{aligned}
& |c|_{S}=\{x \in \omega: x \text { is strongly } S \text {-summable }\}, \\
& \left|c_{0}\right|_{S}=\{x \in \omega: x \text { is strongly } S \text {-summable to } 0\} .
\end{aligned}
$$

We call $|c|_{S} \cap l_{\infty}$ the bounded strong summability field of $S$.

Recall that if $T=\left(t_{n, k}\right)$ is an infinite array of scalars where $k$ and $n$ range over $\mathbb{N}$ and $x, y \in \omega$, we say that $T x=y$ if $\sum_{k=1}^{\infty} t_{n, k} x_{k}=y_{n}$ for all $n \in \mathbb{N}$ and call $T$ a matrix summability method. The matrix $T$ is called nonnegative if $t_{n, k} \geq 0$ for all $n, k \in \mathbb{N}$. A matrix summability method is called regular if $T x \in c$ and $\lim T x=\lim x$ whenever $x \in c$ and a sequence is said to be strongly $T$-summable to $L$ if $\lim _{n} \sum_{k=1}^{\infty} t_{n, k}\left|x_{k}-L\right|=0$.

Given a nonnegative regular matrix $T$, there are a few ways of generating an $R$-type summability method. Set $c_{T}=\{x \in \omega: \lim T x$ exists $\}$ and define $\tau: c_{T} \rightarrow \mathbb{R}$ by $\tau(x)=\lim T x$. It is straightforward to verify that $\tau: c_{T} \rightarrow \mathbb{R}$ is an $R$-type summability method. Observe that $c_{T} \cap l_{\infty},|c|_{T}$, and $|c|_{T} \cap l_{\infty}$ are subspaces of $c_{T}$ and that the restriction of $\tau$ to any of these subspaces is also an $R$-type summability method. Also observe that $\tau$ restricted to $|c|_{T}$ is strong $T$-summability.

A regular nonnegative matrix $T$ can also be used to define "convergence in $T$-density" and " $T$-statistical convergence." Let $A \subseteq \mathbb{N}$ and let $\delta(A)=$ $\lim _{n} \sum_{k=1}^{\infty} t_{n, k} \chi_{A}(k)=1$ when it is defined. We say that a sequence is convergent in $T$-density to $L$ if there is a $B \subseteq \mathbb{N}$ such that $\delta(B)=1$ and $(x-L) \chi_{B} \in c_{0}$ and $T$-statistically convergent to $L$ if $\delta\left(\left\{k:\left|x_{k}-L\right|<\varepsilon\right\}\right)=1$ for all $\varepsilon>0$. Let $D_{T}$ be the collection of all sequences that are convergent in $T$-density and $S_{T}$ be the collection all $T$-statistically convergent sequences. It can be established that $D_{T}\left(S_{T}\right)$ together with the functional that assigns each element of $D_{T}\left(S_{T}\right)$ the value to which the sequence converges in $T$-density (to which the sequence is $T$-statistically convergent to) is an $R$-type summability method.

The preceding $R$-type summability methods have occurred frequently in the literature. If $T$ is the Cesaro matrix, then $\tau$ is convergent in arithmetic mean when it is defined on $c_{T}$ and strong Cesaro summability when it is restricted to $|c|_{T}$. Strong Cesaro summability first made its appearance in the literature in 1913, when Hardy and Littlewood extended Fejer's result that a Fourier series is convergent in arithmetic mean to the result that a Fourier series is strongly Cesaro summable [11]. Strong Cesaro summability also appears as "weakly mixing" in ergodic theory. Moreover, the density arising from the Cesaro matrix 
is called the "natural density" in number theory. The notion of $T$-statistical convergence generated by the Cesaro matrix, which is usually called statistical convergence, was introduced in [8] and has recently been studied in $[6,9,14$, $15,20]$.

$R$-type summability methods are not necessarily generated by matrix methods. Chun and Freedman have given examples of nonmatrix $R$-type summability methods in [4]. Also, Mazur has shown there is a continuous nonnegative linear functional $\nu: l_{\infty} \rightarrow \mathbb{R}$ that satisfies $\nu(x)=\lim x$ for all $x \in c$ and $\nu(x y)=\nu(x) \nu(y)$ for all $x, y \in l_{\infty}[16] . \nu$ is a nonmatrix $R$-type summability method.

\section{CONVERGENCE IN $\mu$-DENSITY AND $\mu$-STATISTICAL CONVERGENCE}

The following definition describes two ways to generate an $R$-type summability method given a finitely additive two-valued measure. As will be noted later, this definition includes the usual definition of convergence as well as the usual definitions of convergence in density and statistical convergence with respect to a nonnegative regular matrix summability method.

Definition 1. Let $\mu$ be a complete $\{0,1\}$-valued finitely additive measure defined on a field $\Gamma$ of subsets of $\mathbb{N}$ that contains all finite subsets of $\mathbb{N}$ and suppose $\mu(A)=0$ if $|A|<\infty$. If $x \in \omega$, we say that

(1) $x$ is $\mu$-density convergent to $L$ if there is an $A \in \Gamma$ such that $(x-L) \chi_{A} \in c_{0}$ and $\mu(A)=1$.

(2) $x$ is $\mu$-statistically convergent to $L$ if $\mu\left(\left\{k:\left|x_{k}-L\right| \geq \varepsilon\right\}\right)=0$ for all $\varepsilon>0$.

It is straightforward to verify that convergence in $\mu$-density and $\mu$-statistical convergence are $R$-type summability methods. Also note that a sequence has a subsequence convergent to $L$ if it is either $\mu$-statistically convergent to $L$ or convergent in $\mu$-density to $L$.

Given an $R$-type summability method, there is a natural measure associated with it. Let $S$ be an $R$-type summability method, $\Gamma=\left\{A \subseteq \mathbb{N}: S\left(\chi_{A}\right)=0\right.$ or 1$\}$ and define $\mu: \Gamma \rightarrow\{0,1\}$ by $\mu(A)=S\left(\chi_{A}\right)$. It can be shown that $\Gamma$ and $\mu$ fulfill the requirements of the preceding definition, that a sequence is $\mu$-statistically convergent to $L$ whenever it is strongly $S$-summable to $L$, and that a bounded sequence is strongly $S$-summable to $L$ if it is convergent in $\mu$-density to $L[7]$.

The following definition and proposition were motivated by [9], where it is shown that a sequence is statistically Cauchy if and only if it is statistically convergent. We also note that if $\mu$ is the measure generated by the usual definition of convergence, then the following definition is equivalent to the usual definition of Cauchy.

Definition 2. Let $x \in \omega$. Then $x$ is $\mu$-Cauchy if for every $\varepsilon>0$ there is an $n \in \mathbb{N}$ such that $\mu\left(\left\{k:\left|x_{k}-x_{n}\right|<\varepsilon\right\}\right)=1$.

Proposition 3. Let $x \in \omega$. Then $x$ is $\mu$-statistically convergent if and only if $x$ is $\mu$-Cauchy.

Proof. First we establish that a $\mu$-statistically convergent sequence is $\mu$-Cauchy. Let $x \in \omega, \varepsilon>0$, and suppose $x$ is $\mu$-statistically convergent to $L$. Since 
$\mu\left(\left\{k:\left|x_{k}-L\right|<\varepsilon / 2\right\}\right)=1$, we can select an $n(\varepsilon) \in \mathbb{N}$ such that $\left|x_{n(\varepsilon)}-L\right|<$ $\varepsilon / 2$. The triangle inequality now yields that $\mu\left(\left\{k:\left|x_{k}-x_{n(\varepsilon)}\right|<\varepsilon\right)\right\}=1$. Since $\varepsilon$ was arbitrary, $x$ is $\mu$-Cauchy.

Now suppose that $x$ is $\mu$-Cauchy. Select $n(1)$ such that

$$
\mu\left(\left\{k:\left|x_{k}-x_{n(1)}\right|<1\right\}\right)=1
$$

and let $A_{1}=\left\{k:\left|x_{k}-x_{n(1)}\right|<1\right\}$. Suppose that $n(1)<n(2)<\cdots<n(p)$ have been selected in such a fashion that if $1 \leq r \leq s \leq p$ and $A_{s}=\left\{k:\left|x_{k}-x_{n(s)}\right|<\right.$ $\left.1 / 2^{s-1}\right\}$, then $\mu\left(A_{r}\right)=1$ and $n(s) \in A_{r}$. Select $N$ such that

$$
\mu\left(\left\{k:\left|x_{k}-x_{N}\right|<1 / 2^{p+1}\right\}\right)=1 .
$$

Since $\mu\left(\bigcap_{1}^{N} A_{j} \cap\left\{k:\left|x_{k}-x_{N}\right|<1 / 2^{p+1}\right\}\right)=1$, there exists an $n(p+1) \in$ $\bigcap_{1}^{N} A_{j} \cap\left\{k:\left|x_{k}-x_{N}\right|<1 / 2^{p+1}\right\}$ such that $n(p)<n(p+1)$ and

$$
A_{p+1}=\left\{k:\left|x_{k}-x_{n(p+1)}\right|<1 / 2^{p}\right\} \supseteq\left\{k:\left|x_{k}-x_{N}\right|<1 / 2^{p+1}\right\} .
$$

Observe that $\mu\left(A_{p+1}\right)=1$ and $n(p+1) \in A_{S}$ for all $s \leq p+1$.

Note that since $\left|x_{n(p)}-x_{n(p+1)}\right|<2^{-p} \quad\left(x_{n(p)}\right)$ is Cauchy, and hence there exists an $L$ such that $\lim _{p} x_{n(p)}=L$. We claim that $x$ is $\mu$-statistically convergent to $L$. Let $\varepsilon>0$ be given and select $p \in \mathbb{N}$ such that $\left|x_{n(p)}-L\right|<\varepsilon / 2$ and $\varepsilon>2^{-p}$. Note that if $\left|x_{k}-L\right| \geq \varepsilon$ then $\left|x_{n(p)}-x_{k}\right|>\varepsilon / 2>2^{1-p}$, and hence $k$ is not an element of $A_{p}$. It follows that $\mu\left(\left\{k:\left|x_{k}-L\right| \geq \varepsilon\right\}\right)=0$ and that $x$ is $\mu$-statistically convergent to $L$.

\section{THe EQUiVAlenCE OF $\mu$-STATISTICAL CONVERGENCE AND CONVERGENCE IN $\mu$-DENSITY}

It becomes natural to wonder when the definitions of convergence in $\mu$ density and $\mu$-statistical convergence coincide. For instance, if an $R$-type summability method generates a measure for which the two definitions are equivalent then the last proposition yields a Cauchy criterion for its bounded strong summability field.

We now introduce some of the structures we wish to explore. Let $S$ be a $R$-type summability method and let $\mathscr{M}=\left|c_{0}\right|_{S} \cap l_{\infty}$. Observe that $\mathscr{M}$ is an ideal of $l_{\infty}$ and $c_{0} \subseteq \mathscr{M} . S$ also generates a filter $\mathscr{F}$ of subsets of $\mathbb{N}$. In particular

$$
\mathscr{F}=\left\{A \subseteq \mathbb{N}: S\left(\chi_{A}\right)=1\right\}=\left\{A \subseteq \mathbb{N}: \chi_{A^{c}} \in \mathscr{M}\right\} .
$$

We can use $\mathscr{F}$ to generate a set corresponding to $S$ in $\mathbb{N}^{*}=\beta \mathbb{N} \backslash \mathbb{N}$. Recall that $\mathbb{N}^{*}$ can be identified with the set of all free ultrafilters on $\mathbb{N}$, and if $W(A)=$ $\{\Omega: \Omega$ is a free ultrafilter, $A \in \Omega\}=\bar{A}^{\beta \mathbb{N}} \cap \mathbb{N}^{*}$, then $\{W(A): A \subseteq \mathbb{N}\}$ is a basis for the topology of $\mathbb{N}^{*}$ [19]. Set $\mathscr{K}=\bigcap\{W(A): A \in \mathscr{F}\} . \mathscr{\mathscr { K }}$ is called the support set of $S$.

We note that if $\mu, \mathscr{F}, \mathscr{M}$, and $\mathscr{K}$ are generated by a given $R$-type summability method $S$, they are different descriptions of the same object and that one can pass from one description to the other. For instance, if $\mathscr{M}$ is an ideal of $l_{\infty}$ that contains $c_{0}$, then $\Gamma=\left\{A \subseteq \mathbb{N}: \chi_{A}\right.$ or $\left.\chi_{A} c \in \mathscr{M}\right\}$ is a field of subsets of $\mathbb{N}$ and the mapping $\mu: \Gamma \rightarrow\{0,1\}$ defined by $\mu(A)=1$ if $\chi_{A} c \in \mathscr{M}$ and $\mu(A)=0$ if $\chi_{A} \in \mathscr{M}$ is a complete finitely additive measure that satisfies the 
criterion of Definition 1. Consequently, one could use $\mathscr{M}$ to define related notions of convergence in density and statistical convergence. Observe that if $\mathscr{M}$ had been the ideal generated by an $R$-type summability method then the measure constructed above is just the measure generated by the $R$-type summability method. Also note that if $\mathscr{F}$ is the filter generated by $S, \Gamma=\{A \subseteq \mathbb{N}: A$ or $\left.A^{c} \in \mathscr{F}\right\}$, and $\mu: \mathscr{F} \rightarrow\{0,1\}$ is defined by $\mu(A)=1$ if $A \in \mathscr{F}$ and $\mu(A)=0$ if $A^{c} \in \mathscr{F}$, then $\Gamma$ and $\mu$, respectively, are the field and measure generated by $S$ as the preceding section. We also record the following observation.

Proposition 4. Any nonempty closed subset of $\mathbb{N}^{*}$ is the support set of an $R$-type summability method.

Proof. Let $\mathscr{H}$ be a nonempty closed subset of $\mathbb{N}^{*}$ and let $\mathscr{F}=\{A \subseteq \mathbb{N}: \mathscr{H} \subseteq$ $W(A)\}$. Since $\mathscr{H} \neq \varnothing, \mathscr{F}$ is a filter. Let $\mu$ be the measure generated by $\mathscr{F}$. Since $\mathscr{H}=\bigcap\{W(A): A \in \mathscr{F}\}=\bigcap\{W(A): \mu(A)=1\}, \mathscr{H}$ is the support set of convergence in $\mu$-density and $\mu$-statistical convergence.

Now let $Q$ and $R$ be two $R$-type summability methods. Write $Q \leq R$ if $|c|_{Q} \subseteq|c|_{R}$ and $Q$ and $R$ are consistent on the bounded summability field of $Q$, i.e., $R(x)=Q(x)$ for all $x \in|c|_{Q} \cap l_{\infty}$. Now let $\mathscr{L}$ be a collection of $R$ type summability methods and suppose $(\mathscr{L}, \leq)$ is a lattice. Let $c_{\mathscr{L}}$ be the set of all bounded sequences for which there is a $Q$ in $\mathscr{L}$ such that $x \in|c|_{Q}$, and define $\lambda(x)=Q(x)$. Note that $\lambda$ is well defined: if $x \in c_{\mathscr{L}}$ and $R$ and $Q$ are $R$-type summability methods with the property that $x \in|c|_{R}$ and $x \in|c|_{Q}$, then $x \in|c|_{R \vee Q}$ and $\lambda(x)=R(x)=Q(x)=(R \vee Q)(x)$. Observations similar to the preceding one can be used to establish that $\lambda$ is an $R$-type summability method. Note that the inclusion requirement occassionally yields the consistency requirement. For instance, if each element of $\mathscr{L}$ is generated by a nonnegative regular matrix method, then the Bounded Consistency Theorem [21, p. 88] yields that it suffices to show $|c|_{Q} \subseteq|c|_{R}$ to show that $Q \leq R$. Chun and Freedman [5] have also established a bounded consistency theorem for the strong summability fields of $R$-type summability methods that includes the preceding observation and can be applied to a broader class of $R$-type summability methods.

Before moving on to the substance of this section, we introduce one more $R$-type summability method. Let $G$ be an infinite subset of $\mathbb{N}$ and suppose $G=\left\{n_{1}, n_{2}, \ldots\right\}$ where $n_{1}<n_{2}<\cdots$ and let $c_{G}=\left\{x \in \omega: \lim _{i} x_{n_{i}}\right.$ exists $\}$. Define $\gamma(x)=\lim _{i} x_{n_{i}}$ for all $x \in c_{G}$. For the obvious reason, $\gamma$ is called the subsequence method generated by $G$. One can establish that a subsequence method is an $R$-type summability method either directly or by showing that it is generated by a regular nonnegative matrix method. We also note that if $\gamma_{1}$, $\gamma_{2}$ are the subsequence methods generated by $G_{1}$ and $G_{2}$, then $\gamma_{1} \leq \gamma_{2}$ if and only if $c_{G_{1}} \subseteq c_{G_{2}}$, which occurs if and only if $\left|G_{2} \backslash G_{1}\right|<\infty$.

If $\mathscr{F}$ is a filter, we can use $\mathscr{F}$ to generate a lattice of subsequence methods. Let $\mathscr{L}=\{\gamma: G \in \mathscr{F}\}$. Note that if $\gamma_{1}, \gamma_{2} \in \mathscr{L}$ are generated by $G_{1}$ and $G_{2}$, then the supremum of $\gamma_{1}$ and $\gamma_{2}$ is the subsequence method generated by $G_{1} \cap G_{2} \in \mathscr{F}$. Also note that if $\mathscr{F}$ is the filter generated by an $R$-type summability method, convergence in $\mu$-density is equivalent to convergence with respect to the lattice of subsequence methods generated by $\mathscr{F}$.

Before giving the promised characterization, we need to state a few more definitions. 
Definition 5 (Additive property for null sets). The measure $\mu$ has the APO if, given a collection of null sets $\left\{A_{j}\right\}_{i \in \mathbb{N}} \subseteq \Gamma$, there exists a collection $\left\{B_{j}\right\}_{i \in \mathbb{N}} \subseteq \Gamma$ with the properties $\left|A_{j} \triangle B_{i}\right|<\infty$ for each $i \in \mathbb{N}, B=\cup_{1}^{\infty} B_{i} \in \Gamma$, and $\mu(B)=0$.

Definition 6. Let $X$ be a topological space and let $\mathscr{H}$ be a closed subset of $X$. Then $\mathscr{H}$ is a $P$-set if $\mathscr{H}$ is in the interior of every $G_{\delta}$ that contains $\mathscr{H}$.

Definition 7. Let $\mathscr{F}$ be a filter. $\mathscr{F}$ has property (A) if given any countable subset $\left\{A_{j}\right\}$ of $\mathscr{F}$, there exists an $A \in \mathscr{F}$ such that $\left|A \backslash A_{i}\right|<\infty$ for each $j$.

We also need to recall a few elementary results that appear in [7]. If

$$
\begin{aligned}
& D_{\mu}=\left\{x \in l_{\infty}: x \text { is convergent in } \mu \text {-density to } 0\right\}, \\
& S_{\mu}=\left\{x \in l_{\infty}: x \text { is } \mu \text {-statistically convergent to } 0\right\},
\end{aligned}
$$

then $D_{\mu}$ and $S_{\mu}$ are ideals in $l_{\infty}, c_{0} \subseteq D_{\mu} \subseteq S_{\mu}$, is the closure of $D_{\mu}$ in $l_{\infty}$. We also recall that if $\mathscr{G}$ is an ideal of $l_{\infty}$ that contains $c_{0}$ and $\mu$ is the measure generated by $\mathscr{G}$, then $D_{\mu} \subseteq \mathscr{G} \subseteq S_{\mu}$. As in [7], the problem of characterizing the measures for which $\mu$-statistical and convergence in $\mu$ density are equivalent is the same problem as characterizing the measures for which $D_{\mu}$ is closed in $l_{\infty}$.

Theorem 8. Let $S$ be an R-type summability method. The following are equivalent:

(a) If $\mu$ is the measure generated by $S$, then $\mu$-statistical convergence and convergence in $\mu$-density are equivalent.

(b) If $\mathscr{M}$ is the ideal generated by $S$, then the closure of $\mathscr{M}$ in $l_{\infty}$ contains no proper dense subideals that contain $c_{0}$.

(c) $\mu$ has the APO.

(d) If $\mathscr{F}$ is the filter generated by $S$, then $\mathscr{F}$ has property (A).

(e) If $\mathscr{K}$ is the support set of $S$, then $\mathscr{K}$ is a P-set.

(f) If $\mathscr{L}$ is the lattice of subsequence methods generated by $S$, then every countable subset of $\mathscr{L}$ has an upper bound in $\mathscr{L}$.

Proof. (a) implies (b). We establish the contrapositive. Let $\mathscr{M}$ be the ideal generated by $S$ and suppose that $\mathscr{G}$ is a dense proper subideal of $\overline{\mathscr{M}}$ that contains $c_{0}$. Since $\overline{\mathscr{M}}$ and $\mathscr{G}$ contain the same set of sequences of 0 's and 1's, they both generate the same measure. Our previous remarks assert that $D_{\mu} \subseteq \mathscr{G} \subseteq \overline{\mathscr{M}} \subseteq S_{\mu}$. Since $\mathscr{G} \neq \overline{\mathscr{M}}$, it follows $D_{\mu} \neq S_{\mu}$.

(b) implies (c). Now we show that $D_{\mu}=S_{\mu}$, then $\mu$ has the APO. Note that if $D_{\mu}=S_{\mu}$ then $D_{\mu}$ is closed. Also note that, via a standard disjointification argument, it suffices to show that APO is satisfied for disjoint collections of null sets. Let $\left\{A_{n}\right\} \subseteq \Gamma$ be a collection of pairwise disjoint null sets. Define $y \in c_{0}$ by $y_{i}=1 / i$ and define $z^{n}$ by $z^{n}=\sum_{i=1}^{n} y_{i} \chi_{A_{i}}$. Note that $\left(z^{n}\right)$ is Cauchy in $l_{\infty}$, hence there is a $z \in l_{\infty}$ such that $\left\|z^{n}-z\right\|$ tends to 0 as $n$ tends to infinity. Note that $z_{k}=1 / i$ if $k \in A_{j}$ and $z_{k}=0$ if $k \notin \bigcup_{1}^{\infty} A_{i}$. Since $D_{\mu}$ is closed, we have that $z \in D_{\mu}$ and hence there exists a $B \in \Gamma$ such that $\mu(B)=0$ and $z \chi_{B} c \in c_{0}$.

Select $N_{1}<N_{2}<\cdots$ such that if $k \geq N_{i}$ and $\left|z_{k}\right| \geq 1 / i$, then $k \in B$. Set $B_{i}=\left\{k: k \in A_{i}, k \geq N_{i}\right\} \cup\left\{k: k \in B, N_{i-1}<k \leq N_{i}\right\}$. It is clear that 
$\left|A_{i} \triangle B_{i}\right|<\infty, B_{i} \subseteq B$, and $B \subseteq \bigcup_{1}^{\infty} B_{i}$ (hence $B=\bigcup_{1}^{\infty} B_{i}$ ). This shows that $\mu$ has the APO.

(c) implies (d). Let $\left\{A_{j}\right\} \subseteq \mathscr{F}$ and set $D_{j}=A_{j}^{c}$. Since $\mu\left(D_{k}\right)=0$ for all $k$ and $\mu$ has the APO, there exists a sequence $\left\{B_{k}\right\}$ of $\mu$-null sets such that, if $B=\bigcup B_{k}$, then $\mu(B)=0$ and $\left|B_{k} \triangle A_{k}\right|<\infty$. Let $A=B^{c}$. Note that $B^{c} \backslash B_{k}^{c}$ is empty for all $k$ and hence $A \backslash A_{k} \subseteq A \backslash\left(D_{k} \cup B_{k}\right)^{c} \subseteq A_{k} \triangle B_{k}$ for all $k$. Since $A_{k} \triangle B_{k}$ is finite, $\mathscr{F}$ has property (A).

(d) implies (a). Suppose $x$ is $\mu$-statistically convergent to 0 and let $A_{n}=$ $\left\{k:\left|z_{k}\right|<1 / n\right\}$. Note that $\mu\left(A_{n}\right)=1$ and hence $A_{n} \in \mathscr{F}$ for all $n$. Pick $A \in \mathscr{F}$ such that $\left|A \backslash A_{n}\right|<\infty$. Observe that $x \chi_{A}$ is a null sequence and hence $x$ is convergent to 0 in $\mu$-density. Since $D_{\mu}=S_{\mu}$, (a) holds.

(d) implies (e). Recall that $\mathscr{K}=\bigcap\{W(A): A \in \mathscr{F}\}$ and suppose that $\mathscr{K} \subseteq$ $\bigcap U_{k}$ where each $U_{k}$ is open in $\mathbb{N}^{*}$. Since $\mathscr{H}$ is compact, there is an $A_{k} \subseteq \mathbb{N}$ such that $\mathscr{K} \subseteq W\left(A_{k}\right) \subseteq U_{k}$ for each $k \in \mathbb{N}$. Suppose $\mathscr{F}$ has property (A), there is an $A \subseteq \mathbb{N}$ such that $\left|A \backslash A_{k}\right|<\infty$ for all $k$. It follows that $\mathscr{K} \subseteq W(A) \subseteq \bigcap W\left(A_{k}\right) \subseteq \bigcap U_{k}$, and hence $\mathscr{K}$ is a $P$-set.

(e) implies (f). Let $\left(\alpha_{k}\right) \subseteq \mathscr{L}$ and suppose $\alpha_{k}$ is generated by $A_{k} \in \mathscr{F}$. Since $\mathscr{K}$ is a $P$-set and $\mathscr{K} \subseteq \bigcap W\left(A_{k}\right)$, there is an open set $U$ such that $\mathscr{K} \subseteq U \subseteq \bigcap W\left(A_{k}\right)$. Now, since $\mathscr{K}$ is compact, we may assume $U=W(A)$ for some $A \subseteq \mathbb{N}$. Using properties of ultrafilters it can be shown that $A \in \mathscr{F}$. Now $W(A) \subseteq W\left(A_{k}\right)$ for each $k$ implies that $\left|A \backslash A_{k}\right|<\infty$ and hence $\alpha_{k} \leq \alpha$ for all $k$.

(f) implies (d). Clear.

The definition of the additive property of null sets was adopted from a similar definition for densities [10] and was given in [7] (where the equivalence of (a), (b), and (c) was established). The support sets of multiplicative summability methods, obtained from matrices and otherwise, have been studied by a number of authors [1, 12, 13, 18]. Atalla, in particular, used property (A) of filters to establish that the support sets of matrices are $P$-sets [1]. Another connection between a (perhaps) stronger additive property of densities and $p$-points has also been established by Mekler [17].

Although the primary intent of the preceding result was to characterize measures for which $\mu$-statistical convergence and convergence in $\mu$-density are equivalent, the equivalence of (b) and (e), in conjuction with Proposition 4, appears to give a new characterization of $P$-sets in $\mathbb{N}^{*}$.

Corollary 9. Let $\mu$ be a measure with the APO. If $\left(x^{r}\right)$ is a countable collection of sequences that are convergent in $\mu$-density, then there exists $\lambda: \mathbb{N} \rightarrow \mathbb{N}$ such that $\lim _{n} x_{\lambda(n)}^{r}$ exists for each $r$ and $\mu(\{\lambda(n): n \in \mathbb{N}\})=1$.

Proof. Let $\mathscr{F}$ be the filter generated by convergence in $\mu$-density. Since each $x^{r}$ is convergent in $\mu$-density, there is an $A^{r} \in \mathscr{F}$ such that $x^{r} \in c_{A^{r}}$. Since $\mathscr{F}$ has property (A), there is an $A \in \mathscr{F}$ such that $\left|A \backslash A^{r}\right|<\infty$ for each $r$, i.e., $c_{A} r \subseteq c_{A}$ for each $r$. Suppose $A=\left\{n_{1}, n_{2}, \ldots\right\}$ where $n_{1}<n_{2}<\cdots$ and $\lambda: \mathbb{N} \rightarrow \mathbb{N}$ satisfies $\lambda(k)=n_{k}$ for all $k$. Now $\lim _{k} x_{\lambda(k)}^{r}$ for each $r$ and $\mu(\{\lambda(k): k \in \mathbb{N}\})=\mu(A)=1$.

Corollary 10. Let $S$ be an $R$-type summability method and let $\mu$ be the measure associated with $S$. If $\mu$ has the APO, then $S$ is equivalent to a lattice of subsequence methods on the bounded strong summability field of $S$. 
Proof. Let $\mathscr{L}$ be the lattice of subsequence methods generated by the filter generated by $S$. If $x$ is in the bounded strong summability field of $S$, then $x$ is $\mu$-statistically convergent and consequently, since $\mu$ has the APO, convergent in $\mu$-density and summable by $\mathscr{L}$.

Let $T$ be a nonnegative regular summability method and $\mu$ be the measure generated by $T$. Observe that convergence in $T$-density is precisely convergence in $\mu$-density and the support set of strong $T$-summability is

$$
\bigcap\left\{W(A): A \subseteq \mathbb{N}, \lim _{n} \sum_{k=1}^{\infty} t_{n, k} \chi_{A}(k)=1\right\} .
$$

Proposition 3.2 of [10] now shows $\mu$ has the APO. A similar proof that $\mu$ has the APO can also be found in [7]. Alternatively, Henriksen and Isbell [13] have shown that the subset of $\mathbb{N}^{*}$ generated by $\mu$ is a $P$-set and hence, via theorem 8, $\mu$ has the APO. The observation $D_{\mu} \subset\left|c_{0}\right|_{T} \cap l_{\infty} \subseteq S_{\mu}$ now yields that strong $T$-summability, $\mu$-statistical convergence, and convergence in $\mu$-density are equivalent on the bounded sequences.

It also follows that, for a nonnegative regular matrix method $T$, the hypothesis of Corollary 9 can be replaced with "If $\left(x^{r}\right)$ is a countable collection of bounded sequences that are strongly $T$-summable" or "If $\left(x^{r}\right)$ is a countable collection of sequences that are $T$-statistically convergent" and the conclusion holds when $\mu$ is the measure generated by $T$. Similarly, Corollary 10 can be used to show that the bounded strong summability field of a nonnegative regular matrix method can be described as the bounded summability field of a lattice of subsequence methods. Also, Proposition 3 now yields the following Cauchy criterion for bounded strong $T$-summability.

Corollary 11. Let $T$ be a nonnegative regular summability method and $x=\left(x_{k}\right)$ be a bounded sequence. Then $x$ is strongly $T$-summable if and only if for every $\varepsilon>0$ there is a $n(\varepsilon) \in \mathbb{N}$ such that if $A(\varepsilon)=\left\{k:\left|x_{k}-x_{n(\varepsilon)}\right|<\varepsilon\right\}$ then $\lim _{n} \sum_{k=1}^{\infty} t_{n, k} \chi_{A(\varepsilon)}(k)=1$.

In closing, we note that while not every $R$-type summability method is generated by a nonnegative regular matrix method, if often happens that the bounded strong summability field of a nonmatrix RSM coincides with the bounded strong summability field of a nonnegative regular summability matrix. For instance, if $T$ is the Cesaro matrix the sequences that are convergent in $T$-density cannot be given a locally convex FK topology and hence are not the convergence domain of any matrix method [6] yet the bounded sequences that are convergent in $T$-density are precisely the bounded strongly Cesaro summable sequences. Note that Theorem 8 indicates that if we can find a measure that does not have the additive property for null sets, then the bounded summability field of convergence in $\mu$-density is not the bounded strong summability field of a matrix method. An example of such a measure is given in [10]. If we accept the Continuum Hypothesis, it is possible to find $R$-type summability methods that have bounded strong summability fields that are not the bounded strong summability field of a matrix and generate measures with the APO. Atalla [3] has shown, given the Continuum Hypothesis, that there are $P$-sets in $\mathbb{N}^{*}$ that are not the support set of a matrix method. If we let $\mathscr{H}$ be such a set and $\mu$ be the measure constructed in Proposition 4 then, via Theorem $8, \mu$ has the 
APO and the bounded strong summability field of $\mu$-statistical convergence is not the bounded strong summability field of a matrix method.

\section{REFERENCES}

1. R. Atalla, On the multiplicative behaviour of regular matrices, Proc. Amer. Math. Soc. 36 (1970), 437-446.

2. _ On the consistency theorem in matrix summability, Proc. Amer. Math. Soc. 35 (1972), 416-422.

3. _ Regular matrices and P-sets in $\beta N \backslash N$, Proc. Amer. Math. Soc. 37 (1973), 157-161.

4. C. S. Chun and A. R. Freedman, Theorems and examples for R-type summability methods, J. Korean Math. Soc. 25 (1988), 315-324.

5. _,$A$ bounded consistency theorem for strong summabilities, Internat. J. Math. Sci. 12 (1989), 39-46.

6. J. Connor, The statistical and strong p-Cesaro convergence of sequences, Analysis 8 (1988), 47-63.

7. __ Two valued measures and summability, Analysis, 10 (1990), 373-385.

8. H. Fast, Sur la convergence statistique, Colloq. Math. 2 (1951), 241-244.

9. J. Fridy, On statistical convergence, Analysis 5 (1985), 301-313.

10. A. R. Freedman and J. J. Sember, Densities and summability, Pacific J. Math. 95 (1981), 293-305.

11. G. H. Hardy and J. E. Littlewood, Sur la serie de Fourier d' une function a carre sommable, C. R. Acad. Sci. Paris Sér. I Math. 156 (1913), 1307-1309.

12. M, Henriksen, Multiplicative summability methods and the Stone-Cech compactification, Math. Z. 71 (1959), 427-435.

13. M. Henriksen and J. Isbell, Multiplicative summability methods and the Stone-Cech compactification II. Notices Amer. Math. Soc. 11 (1964), 90-91.

14. I. J. Maddox, Statistical convergence in a locally convex space, Math. Proc. Cambridge Philos. Soc. 104 (1988), 141-145.

15. __ A Tauberian theorem for statistical convergence, Math. Proc. Cambridge Philos. Soc. 106 (1989), 277-280.

16. S. Mazur, On the generalized limit of bounded sequences, Colloq. Math. 2 (1951), 173-175.

17. A. Mekler, Finitely additive measures on $\mathbb{N}$ and the additive property, Proc. Amer. Math. Soc. 92 (1984), 439-444.

18. J. Rainwater, Regular matrices with nowhere dense support, Proc. Amer. Math. Soc. 29 (1971), 361.

19. W. Rudin, Homogeneity problems in the theory of Cech compactifications, Duke Math. J. 23 (1956), 409-420.

20. T. Salat, On statistically convergent sequences of real numbers, Math Slovaca 30 (1980), 139-150.

21. A. Wilansky, Summability through functional analysis, North-Holland, Amsterdam, 1984.

Department of Mathematics, Ohio University, Athens, Ohio 45701

E-mail address: connor@oucsace.cs.ohiou.eds 